\title{
ON THE CHARACTERISTIC ROOTS OF REAL MATRICES
}

\author{
H. H. SCHAEFER
}

Abstract. If $A$ is a real $n \times n$-matrix whose absolute $|A|$ has spectral radius 1 , and if $\epsilon$ is a unimodular characteristic value of $A$, then all odd (respectively, even) powers of $\epsilon$ are characteristic values of $A$ (respectively, of $|A|$ ). In particular, such $\epsilon$ must be a $k$ th root of unity for some $k, 1 \leqq k \leqq 2 n$.

Since the theory of nonnegative square matrices is well developed (cf. [1], [2], [4]), it appears to be desirable to extend some of its methods and results to larger classes of matrices; it is the purpose of this note to indicate such an extension. The idea of utilizing properties of the matrix $|A|=\left(\left|a_{i j}\right|\right)$ to obtain information on $A=\left(a_{i j}\right)$ can be adapted to operator-theoretic settings (such as the one considered by Rota [3]) but doing this in the desired generality requires technical elaboration, and we hope to publish detailed results elsewhere. On the other hand, the case of finite square matrices is of independent interest.

We use the notation and terminology of [2]. Throughout this note, $A=\left(a_{i j}\right)$ denotes a real $n$-square matrix. We write $|A|=\left(\left|a_{i j}\right|\right)$, and $|x|=\left(\left|x_{i}\right|\right)$ for any (complex) $n$-vector $x=\left(x_{i}\right)$. Sp $A$ denotes the set of characteristic roots (spectrum) of $A$, and $r(A)$ denotes the spectral radius (largest of the moduli of the characteristic roots) of $A$.

THEOREM. Let $A$ be a real $n$-square matrix satisfying $r(|A|)=1 .^{1}$ If $\epsilon \in \mathrm{Sp} A$ and $|\epsilon|=1$, then $\epsilon^{2 k+1} \in \mathrm{Sp} A$ and $\epsilon^{2 k} \in \mathrm{Sp}|A|$ for all integers $k$.

Proof. Obviously the assertion holds for $n=1$. Proceeding by induction on $n$, we can suppose that $A$ is indecomposable $[2, \mathrm{p} .122]$, and $n \geqq 2$. If $\epsilon \in \operatorname{Sp} A,|\epsilon|=1$, then $\epsilon x=A x$ for some $n$-vector $x$ $=\left(x_{i}\right) \neq 0$. It follows that $|x| \leqq|A||x|$, hence $|x|=|A||x|$ and $|x|>0$, since $|A|$ is indecomposable and $r(|A|)=1$. Let $\eta_{i}=\operatorname{sgn} x_{i}$, $\eta_{i j}=\operatorname{sgn} a_{i j}, r_{i}=\left|x_{i}\right|>0(i, j=1, \cdots, n)$. We obtain the equations

$$
\epsilon \eta_{i} r_{i}=\sum_{j=1}^{n}\left|a_{i j}\right| r_{j} \eta_{i j} \eta_{j},
$$

Received by the editors October 10, 1969.

AMS 1970 subject classifications. Primary 15A18; Secondary 15A48.

Key words and phrases. $n \times n$-matrix, characteristic value, unimodular spectrum.

1 Unless $|A|$ is nilpotent, $r(|A|)=1$ is a normalization. We shall use it for convenience of formulation. 


$$
r_{i}=\sum_{j=1}^{n}\left|a_{i j}\right| r_{j}
$$

for $i=1, \cdots, n$. Multiplication of (1) by $\left(\epsilon \eta_{i}\right)^{-1}$ and comparison with (2) yields $\epsilon^{-1} \eta_{i}^{-1} \eta_{i j} \eta_{j}=1$ whenever $a_{i j} \neq 0$. Since $\eta_{i j}= \pm 1$, it follows that $x_{(k)}=\left(r_{i} \eta_{i}^{2 k+1}\right)$ is a characteristic vector of $A$ belonging to $\epsilon^{2 k+1}$, and $y_{(k)}=\left(r_{i} \eta_{i}^{2 k}\right)$ is a characteristic vector of $|A|$ belonging to $\epsilon^{2 k}$.

The first of the following corollaries is well known.

CoRollaRy 1. If $A \geqq 0$ satisfies $r(A)=1$, the unimodular spectrum of $A$ is a union of groups of roots of unity.

Corollary 2. Let $A$ be real, $n$-square and satisfy $r(A)=1$. If $\epsilon \in \mathrm{Sp} A$ is unimodular but not a kth root of unity for any $k \leqq 2 n$, then $r(|A|)>1$.

In the sequel we denote by $G_{m}$ the group of all $m$ th roots of unity.

Corollary 3. Let $A$ be real, $n$-square and indecomposable, and suppose that $r(A)=r(|A|)=1$. Then for some $m, 1 \leqq m \leqq n$, and every unimodular characteristic root $\epsilon$ of $A$, one has $\epsilon^{2} \in G_{m}$ and the unimodular spectrum of $A$ is the coset $\epsilon G_{m}$.

To prove Corollary 3 , we note that a theorem of $\mathrm{H}$. Wielandt [4] asserts that in the present circumstances $\epsilon^{-1} A$ is similar to $|A|$ ([2, II.5.7.5, p. 126]; for the proof, see [4, p. 645]). Since $|A|$ is equally indecomposable, the unimodular spectrum of $|A|$ is some $G_{m}, 1 \leqq m \leqq n$. Finally, $\epsilon^{2} \in G_{m}$ by the theorem above. (Remark: The simple example $A=\left(\begin{array}{rr}0 & 1 \\ -1 & 0\end{array}\right)$ shows that, in general, $\epsilon$ is a primitive $2 n$th root of unity.)

Analogous results can be obtained for real matrices $A$ satisfying $r\left(A^{k}\right)=r\left(\left|A^{k}\right|\right)$ for some integer $k>1$; this includes the powerpositive matrices introduced by A. Brauer [1].

\section{REFERENCES}

1. A. Brauer, On the characteristic roots of non-negative matrices, Proc. Advanced Seminar Recent Advances in Matrix Theory (Math. Res. Center U. S. Army, University of Wisconsin, Madison, Wis., 1963), Univ. of Wisconsin Press, Madison, Wis., 1964, pp. 3-38. MR 29 \#5835.

2. M. Marcus and H. Minc, $A$ survey of matrix theory and matrix inequalities, Allyn and Bacon, Boston, Mass., 1964. MR 29 \#112.

3. G.-C. Rota, On the eigenvalues of positive operators, Bull. Amer. Math. Soc. 67 (1961), 556-558; addendum 68 (1962), 49. MR 24 \#A1621.

4. H. Wielandt, Unzerlegbare, nicht negative Matrizen, Math. Z. 52 (1950), 642648. MR 11, 710.

University of TüBINGen, TüBINGen, Germany 